Conclusion Placental examination serves several vital roles in babies born with suspected HIE: it defines pathophysiology, provides important prognostic information regarding future neurodevelopmental outcome, and shows mitigating factors of medicolegal relevance to causation of brain injury. Intrapartum infection and chorioamnionitis are associated with poor neonatal outcomes including cerebral palsy. Only 30\% placentas were examined in our tertiary centres, yet those examinations showed a high incidence of chorioamnionitis. The low rate of placentas being submitted for examination in neonates born depressed, coupled with the high incidence of proven chorioamnionitis in those submitted, is of great concern.

\section{PP.43 PERINATAL OUTCOMES AND TRAVEL TIME TO MATERNITY SERVICES: ANALYSIS OF BIRTH OUTCOME DATA IN WALES FROM 1995 TO 2009}

doi:10.1136/archdischild-2013-303966.323

'S Paranjothy, 'WJ Watkins, 'K Rolfe, 'Y Gong, ${ }^{2} \mathrm{R}$ Adappa, 'F Dunstan, 'S Kotecha. ${ }^{\prime}$ Cardiff University, Cardiff, UK; ${ }^{2}$ Cardiff and Vale University Health Board, Cardiff, UK

Objective To study the association between travel time from home to hospital on intrapartum stillbirth and neonatal mortality.

Population All births to women who were resident in Wales between $1995-2009(n=498,052)$.

Outcome Measures Intrapartum stillbirth, early and late neonatal mortality.

Methods We calculated the travel time to all hospitals with maternity services based on the grid reference for postcode of mother's place of residence at the time of birth. We used logistic regression to obtain odds ratios for the association between travel time and outcome, adjusted for maternal age, parity, Townsend score for social deprivation and urban/rural location.

Results There were 412,827 singleton births during the study period. The intrapartum stillbirth rate was 0.3 per $1,000(n=135)$; early neonatal death rate 1.5 per $1,000(n=609)$ and late neonatal death rate 0.6 per $1,000(n=251)$. The median travel time to place of birth was 17 minutes IOR $(11,27)$, and the median distance travelled was $11.7 \mathrm{~km}$. The risk of early neonatal death increased with travel time of at least 45 minutes to place of birth (adjusted OR 1.7 $95 \%$ CI 1.2, 2.3). In order to explore whether or not birth outcomes were associated with location of maternity services we repeated the analysis using travel time from home to nearest hospital with maternity services and found no association.

Conclusion Although the risk of adverse birth outcomes is increased with longer travel times to the place of birth this is not explained by distance to the nearest hospital with maternity services.

\section{PP.44 STRESS IN EARLY PREGNANCY IN THE AETIOLOGY OF GASTROSCHISIS: AN INCIDENT CASE CONTROL STUDY}

doi:10.1136/archdischild-2013-303966.324

\section{A Evans, S Paranjothy, S Palmer. Cardiff University, Cardiff, UK}

Background Maternal stress is associated with increased risk of spina bifida and anencephaly ${ }^{1}$. We investigated the effect of major stressful life events in the first trimester on risk of gastroschisis, accounting for the mediatory effects of social support and known risk factors such as cigarette smoking, low body mass index and poor nutrition.

Method We analysed data from an incident case control study of pregnant women resident in five UK regions between 01.07.2007 and 28.02.2010. Three controls were recruited for each case. Major stressful life events and social support were assessed using questions from several validated assessment tools, during interviews in the antenatal period. Logistic regression was used to obtain odds ratios for the association between maternal stress and risk of gastroschisis.

Results During the study period, 124 gastroschisis cases were identified by collaborating centres. $73 \%$ of cases $(n=91)$ and $70 \%$ of controls $(n=217)$ were recruited. In the multivariable model including social class of the mother, cigarette smoking, alcohol consumption, body mass index, folic acid and fruit and vegetable consumption, major stressful life events had an independent effect on the risk of gastroschisis (aOR 4.9 95\% CI 1.2.19.4). Moving house in first trimester was also an independent risk factor (aOR 4.9 95\% CI 1.7.13.9). Lack of social support was found to be a partial mediator for stress.

Conclusion These findings provide new evidence that maternal stress plays a role in the aetiology of gastroschisis, possibly through increased production of corticosteroids that have been shown to be teratogenic in animal models.

\section{REFERENCE}

1. Carmichael SL, Shaw GM, Yang W, et al, Maternal stressful life events and risks of birth defects. Epidemiology 2007:18:356-361.

\section{PP.45 PRETERM CAESAREAN SECTION: THE IMPLICATIONS FOR FUTURE OBSTETRIC CARE}

doi:10.1136/archdischild-2013-303966.325

NB Bozreiba, SC Cooley, SCS Coulter Smith. Royal college of physician Ireland, Dublin, Ireland

Introduction Preterm birth is the leading cause of neonatal morbidity and is associated with increased rates of operative delivery. Little is known about the impact of preterm caesarean section on mode of delivery and outcome in subsequent pregnancies.

Aim To determine the impact of preterm caesarean section in primips and multips on mode of delivery and obstetric outcome in subsequent pregnancies.

Study design We designed a retrospective review of all deliveries in the Rotunda Hospital from January $1^{\text {st }} 2000$ to December $31^{\text {st }} 2005$.

All preterm deliveries (less than 37 weeks gestation) were identified and those requiring caesarean delivery formed the study cohort. All cases with previous operative deliveries were excluded and the remaining cases were reviewed for outcome in subsequent pregnancies.

Results There were 879 preterm caesarean sections during the study period representing $6.6 \%$ of all sections over the study period (879/13336).

In total $672(76.4 \%)$ met the inclusion criteria and of these 408 $(60.8 \%)$ went on to have a further delivery in the hospital. Preterm caesarean section was associated with a vaginal delivery rate of 32 to 44 percent in subsequent pregnancies. There is associated increased neonatal morbidity contributed to primarily by the high incidence of preterm birth in subsequent pregnancies (22.34\%). There were also 3 neonatal deaths in subsequent pregnancies in the cohort.

The overall classical caesarean section rate was six percent and there was one caesarean hysterectomy in our cohort.

Conclusions Preterm caesarean section is associated with adverse fetal outcome in subsequent pregnancies.

\section{PP.46 WHAT INFLUENCES A PARENT'S DECISION-MAKING FOR PERINATAL AUTOPSY? A QUALITATIVE INVESTIGATION}

doi:10.1136/archdischild-2013-303966.326

${ }^{1}$ S Meaney, ${ }^{2}$ S Gallagher, ${ }^{1} \mathrm{JE}$ Lutomski, ${ }^{3} \mathrm{~K}$ O' Donoghue. ' National Perinatal Epidemiology Centre, University College Cork, Ireland; ' ${ }^{2}$ ept. of Psychology, University of Limerick, Ireland; ${ }^{3}$ Dept. of Obstetrics and Gynaecology, University College Cork, Ireland

Objectives This study aims to gain insight into parents' perception of autopsy and the decision-making processes. 